\title{
Positioning with Bluetooth
}

\author{
Josef Hallberg, Marcus Nilsson, Kåre Synnes \\ Luleå University of Technology / Centre for Distance-spanning Technology \\ Department of Computer Science and Electrical Engineering \\ 97187 Luleå, Sweden. \\ \{Josef.Hallberg, Marcus.Nilsson, Kåre Synnes\}@ cdt.luth.se
}

\begin{abstract}
This paper presents an evaluation of Bluetooth positioning in a general positioning platform. Proceeding the evaluation a Bluetooth based positioning system was implemented in order to complement the theoretical evaluation with empirical tests.

Three different ways of positioning with Bluetooth have been developed. With a registered positioning service a Bluetooth device has an active role in the positioning task as it sends a position on request. A Bluetooth device can also take a more passive role in a positioning task, where the unique address of the device is used by a connected device to look up respective position in a database. It is also possible to forward a position gained from the positioning platform using the peer to peer characteristics in Bluetooth.

This paper does also contain a discussion on the theoretical time requirements for a positioning system based on Bluetooth. Empirical tests show that these requirements hold.
\end{abstract}

\section{Introduction}

Location awareness create possibilities for new applications on different devices. Today we see Bluetooth [1] being integrated on a variety of devices. On some of these the only technology available for position tasks is Bluetooth, on others a cooperation between other technologies and Bluetooth would give an advantage in position tasks.

As many Bluetooth devices as possible should be associated with a position in order to get good position source coverage. In order to utilize surrounding Bluetooth devices for positioning a Bluetooth positioning server needs to be mobile and the position should change automatically as the device is moved. With the use of a platform integrating several positioning system like Alipes [2] this is possible. Any of the integrated positioning systems can update the position in the platform, which will be sent to Bluetooth positioning clients using the peer to peer characteristics in Bluetooth. To further improve position source coverage computers using Bluetooth for other tasks than positioning can be used.

0-7803-7661-7/03/\$17.00@2003 IEEE
An office computer using Bluetooth to connect a wireless keyboard and mouse can be turned into a position source without the need of any added software.

\section{Positioning}

In this project, we have developed two different methods for the positioning task. The direct method, where the Bluetooth device must be programmed to handle the positioning task, and the indirect method, where no programming is needed. If more than one position is collected from either method in a single run, triangulation is done in order to get a more accurate position. The flowchart of the positioning task can be seen in figure 1

With the direct method the device can operate standalone and do not need any kind of connection to other services or devices. The client asks the Bluetooth device if it has a positioning service. If the Bluetooth device answers positively on this request the client will connect to this service to retrieve a position. If the answer is negative the client will fall back to the indirect method. The indirect method was developed to make it possible to use devices that are not programmable or not capable to incorporate the positioning server. With this method the client will look at the unique address of the communicating device and use this address as a key to lookup the position in a database. The server for this database, which we call a location server, can run locally or on a different computer on the network. By having both these methods we have made it possible to use almost every Bluetooth device as a positioning source, without compromising the mobility aspect or requiring specialized hardware.

Both of these methods can of course be used with dynamic positions, such as in a mobile unit. The only criteria is that the device must be able to change the position locally or in the positioning server. However we believe that the Bluetooth positioning service is the one that is best suited for mobile units. This method does not require any other connection than the connection between the position server and the receiver. The position in a mobile unit can be received from any other positioning source such as GPS or another Bluetooth positioning device. 


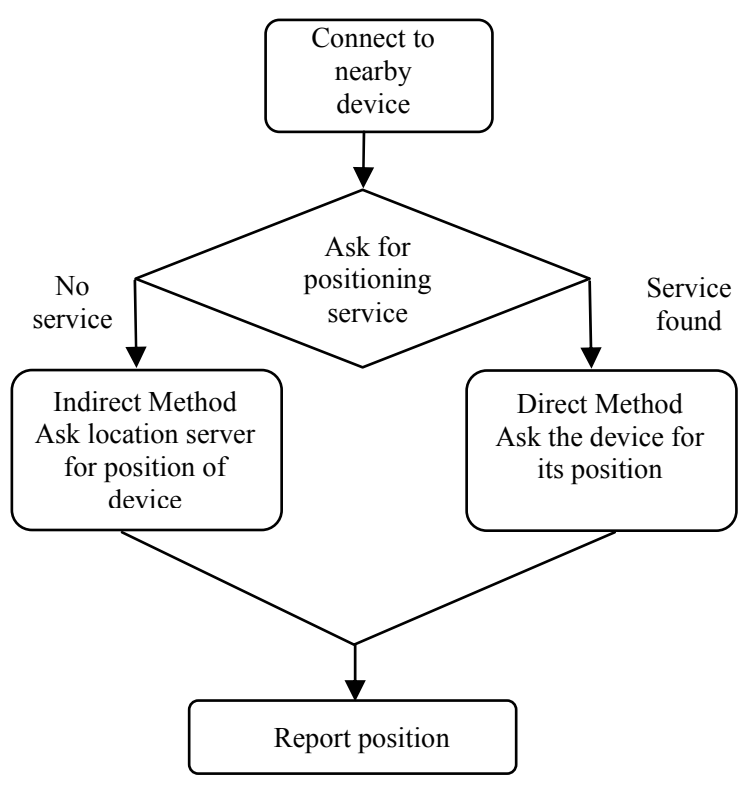

Figure 1: Flowchart for positioning task

\subsection{Position a moving target}

The time ( $\mathrm{t}$ ) in seconds that it takes to travel through a circle passing the center with a diameter $(d)$ at a speed of $s$ is given by:

$$
t=d / s
$$

If we assume that a person has an average walking speed of $1.2 \mathrm{~m} / \mathrm{s}$ and that the circle around the Bluetooth position source where the signal strength is high enough has $20 \mathrm{~m}$ in diameter then the time for traveling through this circle would be $16.7 \mathrm{~s}$. This mean that in order to receive a position from a Bluetooth positioning server the whole process must not take more that $16.7 \mathrm{~s}$ to be successful. If we also consider a Bluetooth device is not constantly looking for new devices then this time will decrease even more. The time between device scans must be at least $1.28 \mathrm{~s}$ and will therefore give us a worst case of $15.4 \mathrm{~s}$ for the maximum time for a position retrieval. This is of course only applicable if we are looking at only one positioning server. When more position servers are present at the same time we must consider the possibility that a new device can become reachable when communication with other devices is ongoing. If this communication lasts longer then the time between device scans the maximum time allowed for a successful position retrieval will decrease even more.

\section{Theoretical evaluation}

How well does Bluetooth work as a source for positions? Let us look at how Bluetooth can work within the important aspects for radio based positioning in a mobile context.

\subsection{Security}

If people will exchange their position with others it is very important that this information is received by the right receiver and that it is not possible to eavesdrop on the information exchange [3]. Bluetooth has many security features, both authentication and encryption of the link is incorporated.

\subsection{Physical aspects}

There are a number of aspects to take into consideration when it comes to wireless communication. Such aspects can be regarding range, angle and power consumption. For a positioning task it is also interesting to know what level of accuracy that is to expect from the device. For mobile devices it is also important to conserve power and thus there needs to be a balance between range and power consumption.

Bluetooth has a typical range from $10 \mathrm{~m}$ to $100 \mathrm{~m}$ depending on the power class of the device. At current date class 3 , with a typical $10 \mathrm{~m}$ radius is the most common.

Positioning systems are most common in mobile contexts where power is limited and it is therefore important to have systems that conserves power. Bluetooth is designed to consume little power and is suitable for mobile devices. Bluetooth also has different modes for conserving power depending on how the device is used. There is also a possibility to negotiate the transmission power with another device to reach the lowest possible power consumption with remaining connection. However, power consumption is still an issue.

\subsection{Accuracy}

Accuracy in this Bluetooth positioning system is dependent on the maximum range of the system. The longer the range the higher the error can be. This is based on the assumption that only one position is read. However, there are methods of improving the accuracy. Bluetooth for example has point to multi point connectivity and may be able to read several positioning sources at once. The optional received signal strength function in Bluetooth could possibly be used to calculate the distance to the positioning source although signal strength tend to vary a lot if there are obstacles in the way or if there is interference. Combining these two techniques would give a more accurate position than each of them separately.

\subsection{Automation}

The user would not want to obtain the position manually, thus the position retrieval needs to be an automatic process. For this automatic device discovery and automatic service discovery helps. The automatic service discovery checks if the positioning service is present in the discovered device. If there is no such service there is no need to ask for the position and the positioning client should fall back on other 


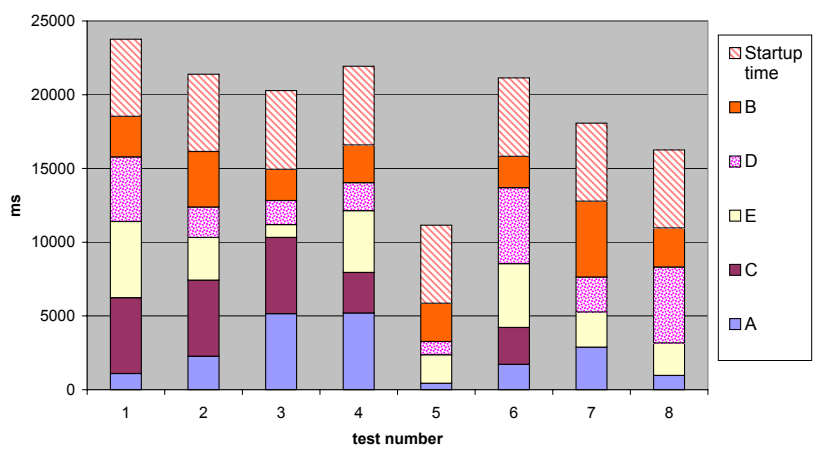

Figure 2: Results from test-runs

methods for positioning. Bluetooth is designed with both device and service discovery and a position task over Bluetooth can therefore be made automatic with ease.

\subsection{Reliability}

For position acquiring it is important that the link is reliable. Packet loss and bit errors have to be handled in some way to make the link reliable, as an unreliable link will increase the time for the position retrieval or even make it fail. Bluetooth works in the ISM band which is a very crowded frequency band. Bluetooth tries to avoid this interference by hopping in frequency 1600 times per second. Bluetooth can detect an error, if it should occur, with Cyclic Redundancy Check and restore corrupted data with Forward Error Correction.

\section{Empirical Tests}

We have investigated the time for a successful position retrieval with non moving clients and position sources. The position sources are a mix of Bluetooth devices running a position service and devices whose unique address is present in the location server locally running at the client. Five devices excluding the client device were placed at different locations in a corridor, see figure 3 . The optional Bluetooth API for signal strength has very low resolution and is therefore very limited in it's usefulness for calculation of distance. Some simple test confirmed that the value was unreliable and no relation between signal strength and distance could be found. Because of this the accuracy was hardcoded to the maximum range of 10 meters.

The test was performed by letting the client search for a position in its environment and the time was recorded for every connection. The resulting position of the triangulation and the total time for the search was reported in the end. Figure 2 shows the result of our test where every time for the test run is a represented by a bar that is divided into sections for the separated devices. We made 8 tests by hand and recorded the time for every connection per test. The startup time in figure 2 is the difference between the sum of the times for every connection and the measured time for the

\begin{tabular}{|c|c|c|c|c|c|}
\hline Device & A & B & C & D & E \\
\hline Failed connections & 2 & 1 & 6 & 1 & 1 \\
\hline
\end{tabular}

Table 1: number of failed connections for every device

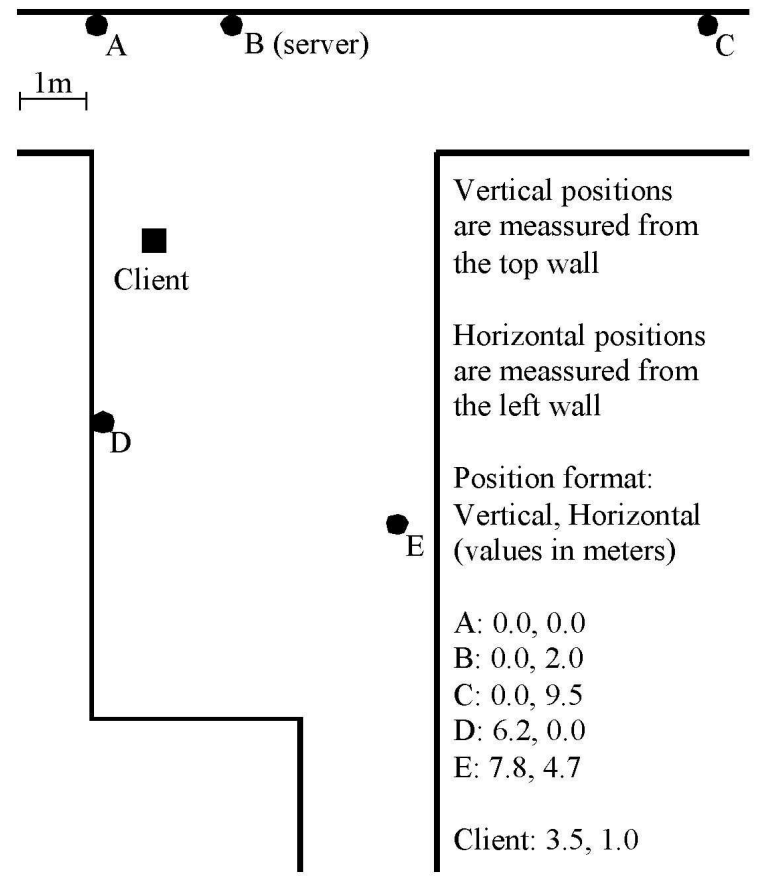

Figure 3: The field-test setup

whole test. We can see that this time is almost the same for every test at an average of $5.3 \mathrm{~s}$. Most of this time is spent searching for nearby devices, and it was set to search for $5 \mathrm{~s}$. The connection to one device will never exceed $5.2 \mathrm{~s}$ as the connection will time out and the client will try to connect to the next device instead.

The amount of failed connections to a device can be seen in table 1 . The average position error is $1.7 \mathrm{~m}+/-1.7 \mathrm{~m}$ based on these few tests. For a more accurate error estimate we need more test results. The lack of a satisfying test environment for a longer period and the fact that we have some possible major performance gains in development for example parallel connections, made us wait with a more complete test. We decided that the results we have will give us sufficient accuracy at this time.

\subsection{Performance evaluation}

The startup time is static and does not change dependent on how many devices we are connecting to. The rest of the time is dependent on the amount of connected devices and the time that every connection takes, see figure 2 . We can calculate that the average time in seconds for a position retrieval in this test was $19.2 \mathrm{~s}$ but the worst case will be

$$
5.3+5.2 * n
$$


Where $\mathrm{n}$ is the amount of devices that are in range. In our test with five devices this would be calculated to $31.3 \mathrm{~s}$

As seen in figure 3, device $\mathrm{C}$ is approximately 10 meter from the client. The Bluetooth devices used in this test are of power-class 3 which have a typical max range of 10 meters. Our test confirms this as we see in table 1 that device $\mathrm{C}$ have very few successful connections. Our hard-coded value for the distance (10 meters) was therefore a good choice. As Bluetooth is a radio based communication there are several things that can disturb the connection between devices. We could see that Bluetooth can be improved in dealing with this as we never did manage to connect to all devices over one test run. Triangulation will in most cases give a better result than 10 meters. Even though all our tests give us a smaller error than 10 meters the theoretical worst case is still 10 meters.

\section{Related work}

Positioning systems have been an area of research for a long time and many different methods and technologies have been used. One of the well known system using techniques for location awareness is the ParcTab [4]. The ParcTab is a rather cheap technology. It uses infrared light for network connection and position information. The downside with the ParcTab system is that it uses specialized hardware for the positioning system and can only give a persons location by which room he or she is in. Another system that have been developed is ORL [5] which is using ultrasonic technology to determine a persons location. This system have a quite good position calculation but suffer from requiring the user to use specialized hardware to retrieve a position. There are also systems like RADAR [6] which like our system uses technology that already is available in mobile units. RADAR share many of the ideas and requirements with us but does not consider that a mobile user can move and the idea of moving position sources. There is also work being done on an official positioning system standard for Bluetooth in the Bluetooth SIG. This standard is under development but is not public. We have not been able to fully understand what differences there are from our work. The little information we have managed to see shows that this standard will require the device to be manufactured for positioning services. If this is the case our implementation can be an interesting extension to the official standard.

\section{Discussion and future work}

The test of our implementation showed that Bluetooth can function as a position transmitter and/or receiver. We also se that our implementation will successfully receive a position if only one position source is present. The worst case for the position retrieval from one source is $10.5 \mathrm{~s}(5.3 \mathrm{~s}$ for the device discovery plus overhead and $5.2 \mathrm{~s}$ for connection to the device). In section 2.1 we concluded that with one position source the maximum time for position retrieval is $15.4 \mathrm{~s} .10 .5 \mathrm{~s}$ is well under $15.4 \mathrm{~s}$ which proves that our implementation will function satisfactory in this scenario.

When more than one position source is present as in our test in section 4 the time for the worst case scenario will exceed 15.4 even after only 2 devices. In this scenario we may get a position dependent on in which order we retrieve the positions and how the different devices are located around the client. This is a result of the fact that the current implementation is not optimized for more than one device and we are expecting great improvements in this area.

One thing that we want to change is that the current implementation only connects to one device at a time. Most of the connection time is spent waiting on the other device and if a parallel connection to all devices could be made this would decrease the total time to the startup time plus the time for the longest connection. For example, test 1 in figure 2 would only take around $10.4 \mathrm{~ms}$ and in that case we would have enough time for positioning when walking as well.

Another interesting improvement would be to let the device maintain connection to the server as long as it is able to. If a device wants to check for a position over and over it would be spared the effort of reconnecting to the known server. This would most likely save both time and power for the device. This however does not only come with advantages but also flaws. There is a limit of seven connections that a server may uphold and if devices were to stay connected it would run out of free connections. This would both hinder new devices from getting a position but it would also hinder the server from doing anything else that it is using the Bluetooth for. A few restrictions could be added in order to limit the downsides of letting devices stay connected to a server. One of these restrictions could be to limit the number of connections allowed for the positioning service. The Server could then use "first in, first out" (FIFO) to supply connections to new devices that wishes to connect.

There would also be a gain in developing a cache system where recently retrieved position that are static can be read locally instead of spending time connecting to that particular device over and over again.

We must also conduct more tests that uses moving position sources and clients to determine the performance of our system in that scenario.

\section{Conclusions}

In this paper we have presented a positioning system for Bluetooth enabled devices. Bluetooth is an inexpensive and small size solution and will probably be integrated in a range of different devices. With a positioning system based on Bluetooth we are able to make these devices location aware. This will open up for new applications and services that can be developed on these devices.

We have showed that the current implementation is fast enough for the task at least for one device. We have also 
showed that future changes will probably make it even faster and by that make it suitable for more devices and in that higher accuracy.

With the use of our location server, even the devices that cannot run any third party software can be used as position sources.

\section{Acknowlegements}

This work was done in the Alipes project and special thanks goes to our sponsors CDT, MRC and SITI. Thanks to James Nord for his work with the Alipes platform.

\section{References}

[1] Jennifer Bray and Charles F Sturman. Bluetooth: Connect Without Cables. Prentice-Hall, Upper Saddle River, 2001.

[2] James Nord, Kåre Synnes, and Peter Parnes. An architecture for location aware applications. In HICSS-35. Hawaii's International Conference on System Sciences, January 2002,

[3] Kåre Synnes, James Nord, and Peter Parnes. Location privacy in the alipes platform. Technical report, Lulea University of Technology/Department of Computer Science and Electric Engineering, 2002.

[4] Bill N. Schilit, Norman Adams, Rich Gold, Michael M. Tso, and Roy Want. The PARCTAB mobile computing system. In Workshop on Workstation Operating Systems, pages 34-39, 1993.

[5] A. Ward, A. Jones, and A. Hopper. A new location technique for the active office, 1997.

[6] V. Bahl and V. N. Padmanabhan. Radar: An in-building rf-based user location and tracking system. In Infocom 2002, Tel-Aviv, Israel, 2000. IEEE Infocom. 